\title{
High-resolution near-IR imaging of the WCd(+OB) environments: pinwheels
}

\author{
Peter G. Tuthill ${ }^{1}$, John D. Monnier ${ }^{2}$, William C. Danchi ${ }^{3}$, \\ and Nils H. Turner ${ }^{4}$ \\ ${ }^{1}$ School of Physics, Sydney University, NSW 2006, Australia \\ ${ }^{2}$ Harvard-Smithsonian Center for Astrophysics, \\ 60 Garden Street, Cambridge, MA 02138, USA \\ ${ }^{3}$ NASA Goddard Space Flight Center, IR Astrophysics, \\ Code 685, Greenbelt, MD 20771, USA \\ ${ }^{4}$ CHARA Array, Mount Wilson Observatory, \\ Mt. Wilson, CA 91203, USA
}

\begin{abstract}
A small number of Wolf-Rayet colliding-wind binaries studied at extremely high angular resolution show elegant dust plumes with an intuitive geometry: that of an Archimedian spiral. A great deal of fundamental information on the binary and the winds is encoded, ultimately teaching us about dust formation and wind-wind collision zones in these fascinating systems. New results are presented summarizing a concerted campaign encompassing a number of systems studied with various techniques over the last five years.
\end{abstract}

\section{Introduction}

The circumstellar environments of colliding-wind binaries (CWB's) have been shown to host much elegant physics, including the recently discovered pinwheel outflows from the Wolf-Rayet colliding wind binaries WR 104 (Ve2-45, WC9d+B0.5V; see Tuthill, Monnier \& Danchi 1999) and WR 98a (IRAS 173803031, WC8-9vd+?; see Monnier, Tuthill \& Danchi 1999; WR catalogue numbers and spectral types from van der Hucht 2001). The census of pinwheels has recently been taken to three with the suspected binary WR 112 (GL 2104, WC9d+OB?; see Monnier et al. 2002a,b) showing evidence for Archimedian spiral structure within a few arcseconds of the core in de-convolved adaptive optics images (Marchenko et al. 2002). The recent 2001 periastron and associated dust formation event for the Rosetta-Stone system WR 140 (HD 193793, WC7pd+O4-5; see Williams et al. 1990) has also yielded rich scientific rewards, with images of the newly-formed dust being carried out in the embedded wind reported in Monnier, Tuthill \& Danchi (2002c).

Although the link between binarity and dust seems clearly established for these systems, the way in which the colliding-winds mediate dust nucleation is uncertain (Williams et al. 1999; Cherchneff et al. 2000). Fortunately, the observational and theoretical pictures of the processes are developing rapidly. Here we present further observational results and discussion of these fascinating systems. 


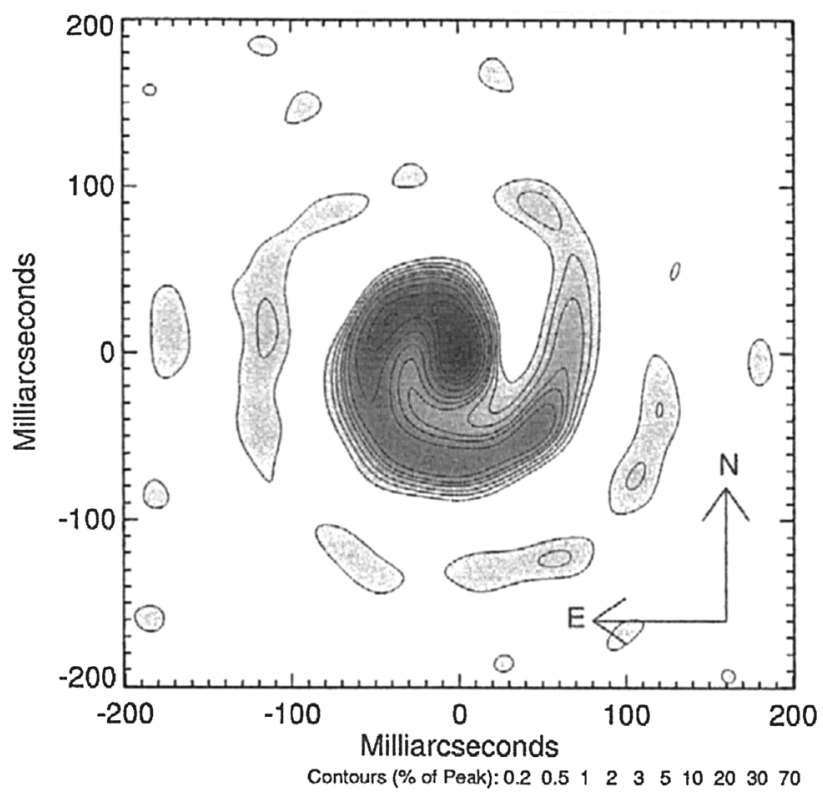

Figure 1. $\quad 2.27 \mu \mathrm{m}$ image of WR 104 (WC9d+B0.5V) reconstructed from data taken in June 1998. This image shows a somewhat wider field and higher dynamic range than previously published images.

\section{Results and discussion}

\subsection{WR 104 (Ve2-45, WC9d+Bo.5V)}

Detailed studies of the time-evolving appearance of the near-perfect Archimedian spiral plume surrounding WR 104 were presented for two epochs in Tuthill et al. (1999) and for six epochs in Tuthill et al. (2002). These utilized aperture masking interferometry (Tuthill et al. 2000) to recover extremely high angular resolution images in the near-infrared with the Keck I telescope. Although there are now new epochs of data showing the further rotation of the spiral structure, these simply confirm the earlier reported morphology and dynamics.

Fully independent confirmation of both the structure and behavior of the plume around WR 104 can now be reported. Recent lunar occultation experiments of Mondal \& Chandrasekhar (2002) showed a resolved, somewhat complex 3 -peaked intensity profile. This was carefully compared with the expected profile from an 'averaged' image from interferometry rotated to the expected angle at the time of the occultation event. Good agreement was found between the profile expected from interpolating the interferometry results, and that deconvolved from the occultation lightcurve. This agreement was quite sensitive to the model conditions, constraining (for example) the rotation rate of the structure to be close to that observed. Chandrasekhar \& Mondal (2002) also reported fine structure within the spiral plume beyond the resolution limit of the Keck observations.

Photometric monitoring of WR 104 over seven years has been reported in Kato et al. (2002), who found that the full-amplitude of variations exceeded 


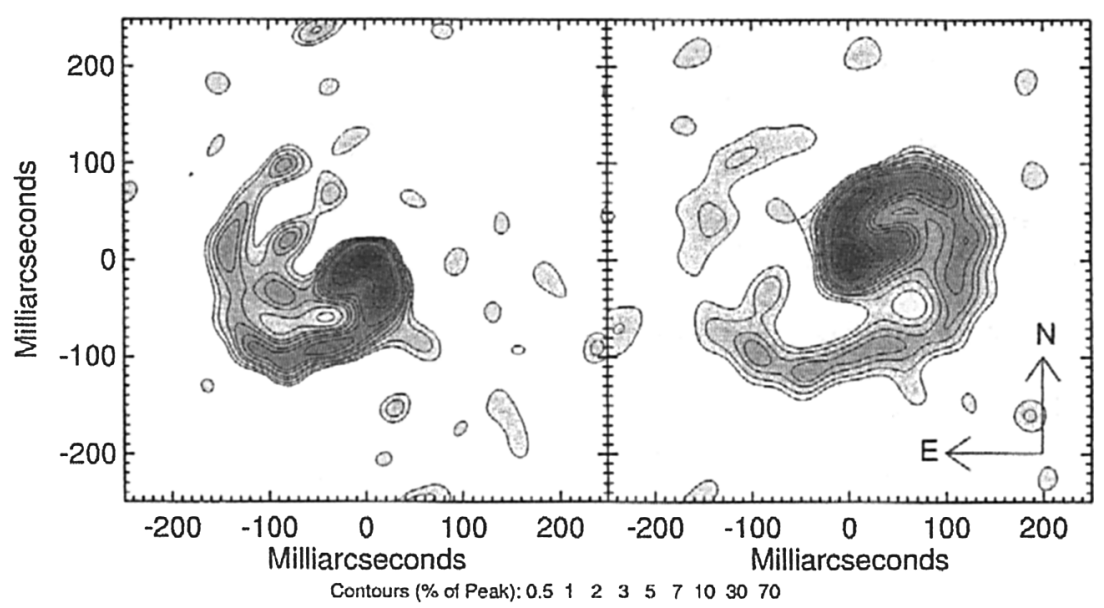

Figure 2. $\quad 2.27 \mu \mathrm{m}$ images of WR 98a (WC8-9vd+?), reconstructed from data taken in September 1998 (left panel) and April 1999 (right panel).

$2.7 \mathrm{mag}$ in $V$. A period analysis of this data showed a number of peaks, with the strongest at $241 \mathrm{~d}$, in close agreement with the best orbital period from interferometry of $243.5 \pm 3 \mathrm{~d}$ (Tuthill et al. 2002). The close-to pole-on viewing angle onto the colliding-wind binary system $\left(i=20^{\circ} \pm 5^{\circ}\right)$ makes this variation in flux more difficult to account for. Plausible scenarios include: $(i)$ variable dust production associated with some eccentricity of the orbit; (ii) phase-dependent obscuration of the central stars by the plume or shock front of the CWB system; (iii) scattered light being modulated as the orientation of the central dusty regions changes; or (iv) anisotropic absorption in the line-of-sight from polar dust clouds which cause a modulation as the spiral structure rotates beneath them.

Incisive studies at the highest resolutions possible will be required to try to make sense of the dust formation in CWB's and its effects on the observable properties. We present, in Figure1, a reconstructed image of WR 104 from aperture masking interferometry (Tuthill et al. 2000) observations made in June 1998. A somewhat wider field-of-view and greater dynamic range is presented than has been previously published, in order to attempt to get the most insight into the detailed morphology of a single snapshot of this pinwheel system.

The first thing to note is, that the spiral plume appears to have little structure across its width, but does exhibit fairly sudden changes along its length. The former observation should not be surprising; the image has structure at the finest scales recoverable given the size of the telescope. Still larger telescopes, or separate element interferometers, will be needed to discern the transverse shape of the dust plume. The apparent changes in surface brightness with length can be accounted for intuitively. After a rapid drop away from the hottest central regions, a fairly extended plateau in surface brightness terminates at around one full rotation. At this point, material in the second and subsequent coils will be eclipsed by material in the first coil, and should therefore cool rapidly. In this frame, we show hints that this second outer coil may be present in the 
data, albeit at low signal-to-noise. Unfortunately, however, there are also artifacts known as grating rings which may mimic (or at least contaminate) this structure, and so the reality of this outer material needs to be carefully checked.

Radiative transfer modelling to the full multi-wavelength data set should go some way towards answering questions about the physical conditions within the dust plume. It is interesting to contrast the overall simplicity of the structures observed with the case of WR 98 a below.

\subsection{WR 98a (IRAS 17380-3031, WC8-9vd+?)}

Imaging of the dust shell around WR 98a has been presented in Monnier et al. (1999). In Figure 2, we show images from two previously reported epochs September 1998 and April 1999. Note that in contrast to the simplicity of the spiral tail in WR 104, here there appear to be more complex and time-evolving features present. Firstly, not one but two bright legs appear to emerge from the brightest region (giving it the appearance of a ' $\mathrm{V}$ '). Further out, the tail structure sometimes appears as a single plume (April 1999) and sometimes with multiple tails or streams (September 1998).

In order to get some intuitive understanding of the possible ways in which our model of the dust plume influences what we might see, we show in Figure 3 geometrical renderings of the expected Archimedian spiral tail. This figure has been constructed from the assumption that dust is formed in the conical wake following the shock front surrounding an OB companion, and furthermore that dust within this cone is highly optically thick.

It is likely, however, that dust may not be optically thick everywhere, particularly as the dust disperses further out into the circumstellar environment. We show in Figure 4 two different possibilities for optically thin plumes: firstly the material has been uniformly distributed throughout the volume of the CWB shock cone, while in the second panel the dust is assumed to exist only at the interface zone between the WR and OB winds. This latter panel is particularly interesting, as the line-of-sight through what is essentially a hollow plume structure generates a number of interesting limb-brightening effects. With structures such as this, if only the very brightest regions are recorded on the map it is possible to get multiple tails, or tails which appear to split or change direction. Detailed studies of geometries such as this may eventually be able to explain the complex behavior of the tail of WR 98a.

\subsection{WR 140 (HD 193793, WC7pd+O4-5)}

One of the most anticipated recent events in the Wolf-Rayet CWB community was the 2001 periastron event of WR 140 . This system produces copious dust at 7.94-yearly intervals coincident with periastron in a highly eccentric $(e=0.8)$ colliding-wind binary (Williams et al. 1990). Monnier Tuthill \& Danchi (2002c) imaged the 2001 event for the first time, and were able to show outward motion of clumps and arc-shaped structures at speeds of around $1.1 \mathrm{mas} / \mathrm{d}$.

The bright dust shell recovered in the images did not, however, conform precisely to the simplest possible models. Naïvely, dust was expected along a crescent-shaped region corresponding to the orbital trajectory of the companion star inflated by the spherically divergent WR wind. Although a one-sided crescent or arc did appear to dominate the recovered images (Monnier et al. 2002c), 


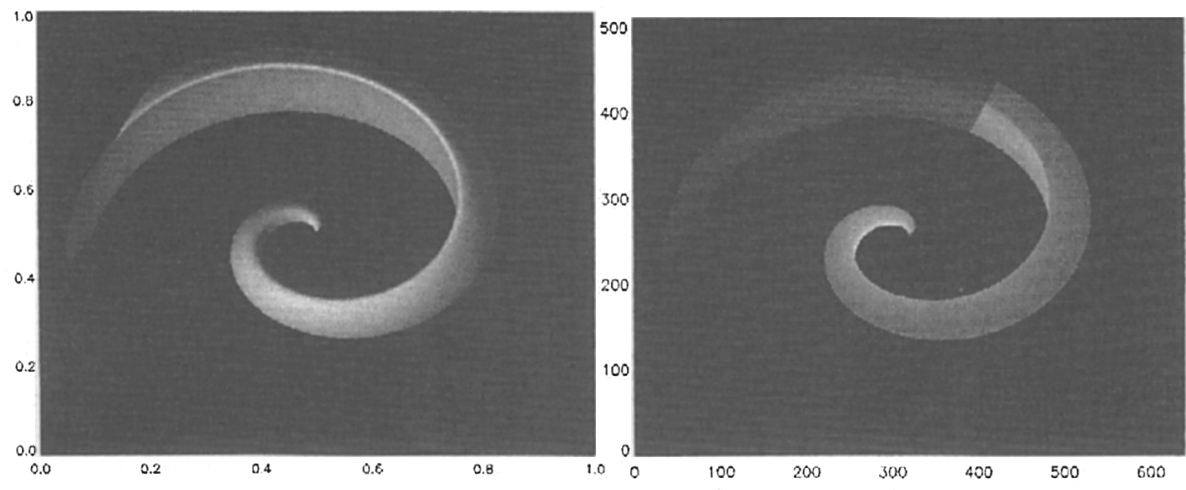

Figure 3. Models of a CWB wind interface region, assuming an opening angle of the shock front of $60^{\circ}$. Left: the (unrealistic) case of such a structure being illuminated from an external source. Right: illumination of an optically thick plume with light from a source located at the center of the system. The shadow on the structure at one turn illustrates the effect of obscuration of outer material by dust closer in.
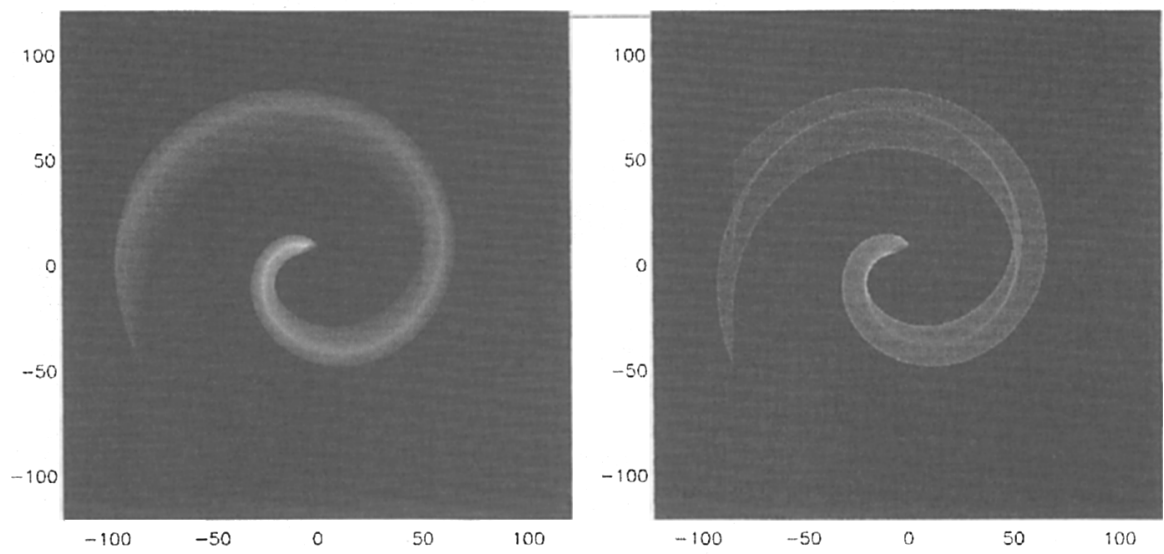

Figure 4. Models of a CWB wind interface region, assuming an opening angle of the shock front of $60^{\circ}$. Left: optically thin dust distributed through the volume of the OB wind. Right: dust only exists at the interface between the OB and WR winds.

additional clumps were apparent, some of which seemed to be on the opposite side of the star entirely from the arc.

In order to investigate the appearance of the dust shell of WR 140 further, we have processed additional data from that previously presented, extending the wavelength coverage to $3.08 \mu \mathrm{m}$. Results are presented in Figure 5. Encouragingly, all structures reported in Monnier et al. (2002c) are independently reproduced. The arc-shaped dust plume seems relatively stronger, as expected, further into the thermal infrared. Furthermore, the complexity of structure, 


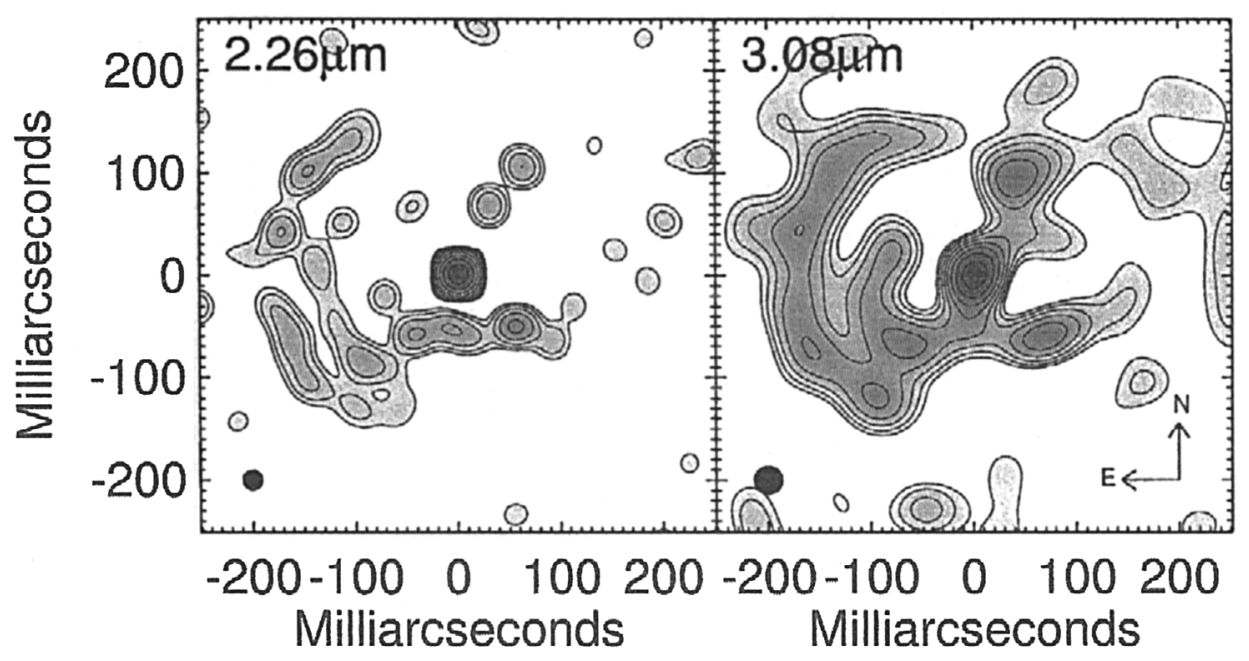

Contours (\% of Peak): $\begin{array}{llllllll}0.5 & 1 & 2 & 4 & 8 & 16 & 32 & 64\end{array}$

Figure 5. $\quad 2.26 \mu \mathrm{m}$ and $3.08 \mu \mathrm{m}$ images of WR 140 (WC7pd+04-5) reconstructed from Keck speckle interferometry data at taken in July 2001.

including the tentative double-shell nature of the arc, and the bright clumps to the South and North-West are also verified.

As discussed earlier, some of this complexity may be caused by line-ofsight effects in viewing an optically thin structure. It is interesting that some clumps not belonging to the obvious arc structure appear on opposite sides of the central point-source. If this displacement is real and not some projection effect, then models of dust formation will need to explain the creation of dust at large distances from the trajectory of the CWB. One possibility may be highly anisotropic winds, in which packets of gas from the OB star may pass like bullets through the WR wind and so trigger dust formation on the opposite side from that expected.

Given the history of episodic dust formation in WR 140, one should expect arcs of dust like the one in Figure 5 to be created every $7.94 \mathrm{yr}$. At an outflow speed of $1.1 \mathrm{mas} / \mathrm{d}$, we should expect structure in the wider circumstellar environment to show periodic shell segments approximately 3 .'2 apart. As these shells would be quite distant from the central stars and therefore quite cool, imaging in scattered light seemed the best option for searching for evidence of past dust formation events.

Figure 6 shows data from the visible adaptive optics experiment on WR 140 at the Maui Space Surveillance Complex. In addition to the bright central star, a faint ( $\Delta V \simeq 8 \mathrm{mag}$ ) companion separated by $4 . \prime 6$ is reported. It is not clear if this is a member of the system, or a field star in apparent association: in either event it will have no influence on the physics of the colliding-wind system.

Despite careful image processing including de-convolution of the PSF of the AO system, no additional extended flux was detected. Specifically, the expected 


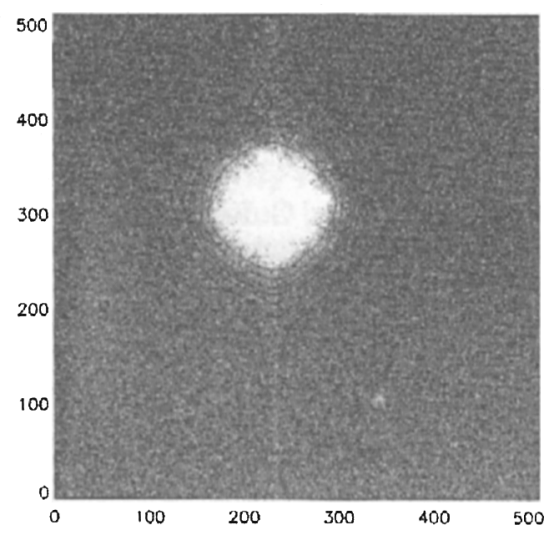

Figure 6. Visible adaptive optics images of WR 140 (WC7pd+O4-5) from the AEOS system on Haleakala, Maui. Data were recorded on 13 March, 2002. The plate scale is $10^{\prime \prime} .24$ on a side. The vertical enhancement of emission passing through WR 140 is due to an instrumental effect known as 'gulching'.

shells at intervals of 3.2 were not seen. The time of observation, shortly after a recent dust formation event, may not be the most fortuitious as it is possible that the newly-formed dust close to the star is shadowing the shells further out, thereby making them harder to image. However, it may be that the older dust shells are dispersed to the extent that specialized techniques, such as deep coronographic imaging, are required in order to see them.

Acknowledgments. We would like to thank Theo ten Brummelaar and Lewis Roberts for the AEOS data. Some data presented herein were obtained at the W.M. Keck Observatory, which is operated as a scientific partnership among the California Institute of Technology, the University of California and the National Aeronautics and Space Administration.

\section{References}

Cherchneff, I., Le Teuff, Y.H., Williams, P.M., Tielens, A.G.G.M. 2000, A\&A 357, 572 Kato, T., Haseda, K., Yamaoka, H., Takamizawa, K. 2002, PASJ (Letters) 54, L51 Marchenko, S.V., Moffat, A.F.J., Vacca, W.D., Côté, S., Doyon, R. 2002, ApJ (Letters) 565 , L59

Mondal, S., Chandrasekhar, T., 2002, MNRAS 334, 143

Monnier, J.D., Tuthill, P.G., Danchi, W.C. 1999, ApJ (Letters) 525, L97

Monnier, J.D., Greenhill, L.J., Tuthill, P.G., Danchi, W.C. 2002a, in: A.F.J. Moffat \& N. St-Louis (eds.), Interacting Winds from Massive Stars, ASP-CS 260, 331

Monnier, J.D., Greenhill, L.J., Tuthill, P.G., Danchi, W.C. 2002b, ApJ 566, 399

Monnier, J.D., Tuthill, P.G., Danchi, W.C. 2002c, ApJ (Letters) 567, L137

Tuthill, P.G., Monnier, J.D., Danchi, W.C. 1999, Nature 398, 487

Tuthill, P.G., Monnier, J.D., Danchi, W.C., Wishnow, E.H., Haniff, C.A. 2000, PASP 112, 555 
Tuthill, P.G., Monnier, J.D., \& Danchi, W.C. 2002, in: A.F.J. Moffat \& N. St-Louis (eds.), Interacting Winds from Massive Stars, ASP-CS 260, 321

van der Hucht, K.A. 2001, New Astron. Reviews 45, 135

Williams, P.M., van der Hucht, K.A., Pollock, A.M.T., Florkowski, D.R., van der Woerd, H., Wamsteker, W.M. 1990, MNRAS 243, 662

Williams, P.M. 1999, in: K.A. van der Hucht, G. Koenigsberger \& P.R.J. Eenens (eds.), Wolf-Rayet Phenomena in Massive Stars and Starburst Galaxies, Proc. IAU Symp. No. 193 (San Francisco: ASP), p. 267

\section{Discussion}

MARSTON: At what distance from WR 98a does the tail of the spiral begin to break up?

TUTHILL: $\sim 100$ mas.

CrowTHER: How do you interpret the partial obscuration of WR 104 that I observed in 1997 with the low incubation you derive for the system?

Tuthill: This is a puzzle. It could be more distant material from the Roche lobe overflow and an envelope ejection event which we suggest preceeded the current phase. However, it is also interesting to note that the variability detected in the light curve at, the same $240 \mathrm{~d}$ period as our orbit also raises questions about material in the line of sight. What is causing changes in $V$ if this system is close to pole-on and the dust is all confined to an equatorial spiral? There may be more complexity to the dust formation than we think.

WILLIAMS: First in response to Crowther's question, WR 104 is not the only WC9 star to show eclipses by line-of-sight dust clumps, so something else may be going on (see Veen, van Genderen, van der Hucht et al. 1998, A\&A 329, 199) . Secondly, a question: from the multi-wavelength images, did you form color and temperature maps?

TUthill: We do have images from 1.65 to $3.08 \mu \mathrm{m}$. However, it is difficult to make color maps as the images are at the limit of the available resolution. Images at $3.08 \mu \mathrm{m}$ show significantly less structure than those at shorter wavelengths, so in order to compare maps at different colors, all maps need to be blurred down to the resolution of the longest-wavelength map. By the time one has done this, most of the clear spiral tail is smoothed out. As an alternate approach, I would suggest trying to model the images with a full radiative transfer code.

KoEnIGSBERger: Are you absolutely sure that the period in WR 104 and WR.98a is the binary period?

TUTHILL: For the two 'pinwheel' systems, a binary seems to be the best explanation. WR 104 has OB absorption lines in its spectrum, and all pinwheel objects have been shown by us to have non-thermal components in their radio spectrum indicative of colliding wind shocks.

CrowTHER: Reply to question by Koenigsberger: dust formation is tough enough for theorists to explain away in the shielded shock cones of $\mathrm{WC}+\mathrm{O}$ binaries. Such shielding would be absent around a single rotating WC star. Observationally, we only see dust formation near periastron in WR 140 . Hence see no credible alternative to binarity in these pinwheels. 
MofFAT: (i) In answer to Owocki's suggestion that WR stars like EZ CMa (WR6, WN4) might produce spirals of matter like those you see: WR 6 shows coherent variations in $P=3.76 \mathrm{~d}$ only, over several cycles; after typically a month, the variation pattern completely changes. This docs not match what you are seeing in the WC9+OB binary WR 104 or other WC dust spirals, that appear to form dust regularly in rotating spirals that repeat cycle after cycle. (ii) The spiral in WR 104 looks relatively smooth, but can you exclude that it, too, would break up into numerous knots and blobs, as seen in WR 140 and other systems? (iii) The key in WR 112 (GL 2104, WC9d+OB?) to detecting the dust spiral was to observe in the mid-IR as opposed to the $K$-band, where one can trace cooler dust out to larger distances from the central binary.

TuTHILL: In answer to your point (ii), we cannot exclude that the spiral tail has internal structure, clumpy or otherwise. The images are currently at the limit of the capability of our $10 \mathrm{~m}$ telescope - interferometers will probably be needed to do a lot better. Interestingly, the lunar occultation observations of WR 104 which I mentioned, were claimed by the authors to suggest fine structure within the spiral tail.

WALBORN: With regard to the question whether the observed periods are orbital, doesn't any of these systems have a radial-velocity period? Such verification in even one case would be persuasive and should be pursued even if difficult.

VAN DER HUCHT: WR 140 (HD 193793, WC7pd+O4-5) has a radial-velocity solution, see Williams, van der Hucht, Pollock, et al. (1990, MNRAS 243, 662) and Setia Gunawan, van der Hucht, Williams et al. (2001, A\&A 376, 460).

TUTHILl: As said, the long-period systems WR 140 has a spectroscopic orbit. The shorter period systems such as WR 98a or WR 104 are difficult, as they are faint in the visible, the lines are broad and the experiments require lots of telescope time. For WR 104, of course, it is further complicated as it appears almost face-on.

HANS ZINNECKER: What is the contrast in your interferometric images?

TUTHILl: Depending on the quality of the data, the dynamic range in the images is usually $100: 1$ or $200: 1$. This makes it difficult to follow dust as it cools and is carried further from the star. 